\title{
FATORES DE RISCO DA ENXAQUECA NA FAIXA ETARIA DE 7 A 15 ANOS
}

\author{
DEUSVENIR DE SOUZA CARVALHO * \\ ELIOVA ZUKERMAN ** \\ SUZANMEIRE NEGRO MINATTI HANUCH * \\ CELIO LEVYMAN * \\ JOSE GERALDO CAMARGO LIMA ***
}

$O$ diagnóstico de enxaqueca baseia-se essencialmente na anamnese. Tem sido citado na literatura, de forma não sistemática, que vários sintomas referidos por crianças ocorrem mais naquelas com enxaqueca que nas demais. Esses sintomas sâo: distúrbio de sono (DS), dor abdominal recorrente (DAR), cinetose (C), hiperatividade $(H)$, vertigens $(V)$, dores do crescimento (DC), vômitos cíclicos (VC) e

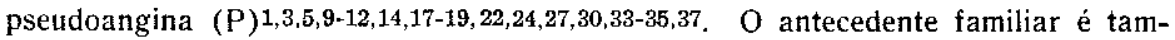
bém considerado um dado importante para o diagnóstico de enxaqueca $2,4,6,8,9,31$. Del Bene 9, que estudou na infância esses sintomas, em conjunto, e o antecedente familiar de cefaléia, denominou-os fatores de risco de cefaléia em crianças. $\mathrm{Na}$ maioria dos estudos esses sintomas são analisados quer isoladamente, quer agrupando número pequeno deles. Esses estudos referem-se alguns a crianças normais, outros a crianças com cefaléia em geral ou enxaqueca e, mesmo, com outras sindromes neurológicas 7,13,15,16, 20,21,23,25,26,28,29.

O objetivo deste trabalho é verificar se esses sintomas (DS, DAR, C, H, $V$, DC, VC, e P) e o antecedente familiar de cefaléia e de enxaqueca, são mais freqüentes em crianças com enxaqueca na faixa etária de 7 a 15 anos e se há diferença quanto ao sexo.

\section{MATERIAL E METODOS}

Separamos dois grupos de crianças denominados A e B. No grupo A foram incluídos 68 pacientes do SITC, com idade de 7 a 15 anos e com diagnóstico de cnxaqueca estiabelecido segundo os critérios de Prensky e Sommer (31), que estabelecem a enxaqueca como cefaléia recorrente, separada por intervalos livres de dor e associada a pelo menos trés dos seguintes achados: $1 \rightarrow$ dor abdominal, náusea ou vômito; 2 - localizaçảo unilateral; 3 - caráter latejante; 4 - alívio após breve período de sono; 5 - aura; 6 - história familiar. No grupo $\mathbf{B}$, foram incluídos 68 alunos de escola pública estadual cuja entrevista com questionário padrăo de perguntas dirigidas, revelou nunca terem cefaléia. O número de entrevistados foi o necessário para ser pareado com o grupo A segundo o sexo e idade. Em ambos os grupos foram colhidos os dados sobre: antecedente familiar de enxaqueca no pai, mae, tios c avós; ocorrência de DS, DAR, C, H, V, DC, VC e $\mathbf{P}$ nos pacientes. Esses sintomas foram assim considerados: distúrbio de sono - ocorrência de sono agitado, sonilóquio, terror noturno, jactatio capitis, bruxismo, sonambulismo e enurese: dor abdominal recorrente - crises recorrentes de dor semelhante a cólica, que aparecem subitamente e duram minutos ou horas, geralmente localizada na região periumbilical e que se irradia por todo o abdome; cinetose - crises de mal estar, palidez, náusea e vômito quando andam em vefculos; hiperatividade - comportamento inadequado,

Disciplina de Neurologia da Escola Paulista de Medicina: * Pós-Graduando em Neurologia Clínica; ** Professor Adjunto, Chefe do Setor de Investigação e Tratamento das Cefaléias (SITC); *** Professor Titular, Chefe da Disclplina. 
com excesso de atividade motora e déficit de atençăo, referido pela familia ou observado por médico; vertigens - episodios recorrentes de tontura rotatória por segundos, acompanhada cventualmente por perspiraçăo, palidez, náusea e vomito, sem perda de consciência ou modificaçåo pela mudança postural; dores do crescimento sensaçōes recorrentes de dor incaracterística não articular e sem fenômenos inflamatórios, que se iniciam à tarde e desaparecem pela manhã; vômitos cíclicos - episódios recorrentes de vômitos espontâneos ou desencadeados ou agravados por odores ou estímulos visuais, como ver outra pessoa vomitando ou ouvir falar sobre o assunto. podendo os vômitos aparecer de forma súbita, persistir por horas ou até alguns dias, levar a desidrataça e traqueza; pseudoangina - dor torácica episódica recorrente, semelhante a angina sem, contudo, ser encontrada qualquer evidencia de anormalidade cardiaca.

Os dados obtidos no grupo A foram comparados aos do grupo B. Os dados referentes aos antecedentes familiares são apresentados em tabela. Consideraram-se sintomas acumulados a ocorrência de DS, DAR, C, H, V, DC, VC e P de forma variadamente associada em cada caso, sendo apresentados em gráfico percentual. A ocorrência dos sintomas concomitantes ou nāo, em cada grupo, por sexo, foi apresentada em tabelas. Os dados especificos da comparação de cada sintoma, por sexo, entre o grupo A e B, foram resumidos em tabela. A análise estátística foi feita pelo teste do $\chi^{2}$ ou Exato de Fisher; para a rejeição da hipótese de nulidade, usou-se valor igual ou menor que $0,05(5 \%)$; quando o valor da estatística calculado apresentou-se significante, usamos um asterisco (s2). Năo foi feita análise estatística quando estudamos a frequiência absoluta e relativa (em percentagem) dos sintomas.

\section{RESULTADOS}

No grupo A todos os 68 pacientes tinham diagnóstico de enxaqueca (enxaqueca comum, $50=75,5 \%$; enxaqueca clássica, $14=20,6 \%$; enxaqueca basilar, $4=5,9 \%$ ). A faixa etária foi dos 7 aos 15 anos, sendo 30 do sexo masculino e 38 do sexo feminino tanto no grupo A como no grupo B; a maioria estava abaixo dos 13 anos. A distribuiçāo por sexo e idade em anos completos consta da tabela 1 . $O$ antecedente familiar de cefaléia em geral no grupo A apareceu em 51 (75\%) e, no grupo B, em $33(48,6 \%)$; o antecedente de enxaqueca apareceu no grupo $A$ em $43(63,2 \%)$ ( no grupo $B$, em $9(13,2 \%)$; houve predominância significante de ambos os antecedentes no grupo A (Tabela 2). A freqüència absoluta e relativa (\%) dos sintomas DS, DAR, C, H, V, DC, VC e $\mathbf{P}$ em cada sexo consta das tabelas 3 e 4 e dos gráficos I

\begin{tabular}{|c|c|c|c|c|c|c|c|c|c|c|}
\hline \multirow{2}{*}{ Sexo } & \multicolumn{9}{|c|}{ Idade (em anos completos) } & \multirow{2}{*}{ Total } \\
\hline & 7 & 8 & 9 & 10 & 11 & 12 & 13 & 14 & 15 & \\
\hline Masculino & 0 & 2 & 7 & 3 & 4 & 10 & 3 & 0 & 1 & 30 \\
\hline Feminino & 3 & 3 & 9 & 4 & 2 & 4 & 10 & 1 & 2 & 38 \\
\hline Total & 3 & 5 & 16 & 7 & 6 & 14 & 13 & 1 & 3 & 68 \\
\hline
\end{tabular}

Tabela 1 - Crianças do grupo $A$ ou $B$, segundo o sexo $e$ idade.

\begin{tabular}{|c|c|c|}
\hline & Grupo A & Grupo B \\
\hline Antecedente de cefaléia & $51 \quad(75,0 \%)^{*}$ & $33(48,6 \%)$ \\
\hline Antecedente de enxaqueca & $43 \quad(63,2 \%)^{*}$ & $9(13,2 \%)$ \\
\hline
\end{tabular}

Tabela $2-$ Antecedente familiar de cefaleia em genal $e$ de enxaqueca nos grupos $\boldsymbol{A} \boldsymbol{E} \boldsymbol{B}$. $* \mathrm{p}<0.05$. 


\begin{tabular}{lrrrr}
\hline & \multicolumn{3}{c}{ Sexo } \\
\cline { 3 - 6 } Sintoma & \multicolumn{2}{c}{ masculino } & \multicolumn{2}{c}{ feminino } \\
& & & $\%$ & $n$ \\
\hline DS & 12 & 40,0 & 15 & 39,4 \\
DAR & 14 & 46,6 & 20 & 52,6 \\
C & 13 & 43,3 & 16 & 42,1 \\
H & 8 & 26,6 & 9 & 23,6 \\
V & 10 & 33,3 & 8 & 21,0 \\
DC & 7 & 23,3 & 15 & 39,4 \\
VC & 7 & 23,3 & 8 & 21,0 \\
P & 4 & 13,3 & 0 & 00,0 \\
Nenhum & 3 & 10,0 & 4 & 10,5 \\
\hline
\end{tabular}

Tabela 3 - Freqüencia absoluta $(n)$ e relativa $(\%)$ dos sintomas, concomitantes ou ndo, em relagão ao sexo (masculino, 30 ; feminino, 38) no grupo $A$. Legenda: $D S$, distúrbio do sono; $D A R$, dor abdominal recorrente; $C$, cinetose; $H$, hiperatividade; $V$, vertigens; $D C$, dores de crescimento; VC, vomitos ciclicos; $P$, pseudoangina.

\begin{tabular}{lrrrr}
\hline & \multicolumn{4}{c}{ Sexo } \\
\cline { 2 - 5 } Sintoma & \multicolumn{2}{c}{ masculino } & \multicolumn{2}{c}{ feminino } \\
& $\mathrm{n}$ & $\%$ & \multicolumn{1}{c}{$\mathrm{n}$} & $\%$ \\
\hline \hline DS & 3 & 10,0 & 19 & 50,0 \\
DAR & 0 & 0,0 & 1 & 2,6 \\
C & 4 & 13,3 & 2 & 21,0 \\
H & 2 & 6,6 & 2 & 5,2 \\
V & 0 & 0,0 & 3 & 7,8 \\
DC & 2 & 6,6 & 1 & 2,6 \\
VC & 1 & 3,3 & 1 & 2,6 \\
P & 1 & 3,3 & 12 & 31,5 \\
Nenhum & 20 & 66,6 & & 2 \\
\hline
\end{tabular}

Tabela 4 - Freqüencia absoluta $(n)$ e relativa (\%) dos sintomas, concomitantes on năo, em relacdo ao sexo (masculino, 30; feminino, 38) no grupo $B$. Legenda: $D S$, distúrbio do sono; $D A R$, dor abdominal recorrente; $C$, cinetose; $H$, hiperatividade; $V$, vertigens; $D C$, dores de crescimento; $V C$, vomitos ciclicos; $P$, psewdoangina.

e Il (Fig. 1), A análise estatística, quanto ao sexo, demonstrou predominancia no sexo masculino de $\mathbf{P}$ no grupo $\mathbf{A}$ e ausência dos sintomas no grupo $B$; há predominância no sexo feminino de $\mathrm{DS}$ no grupo $\mathrm{B}$ (Tabela 5). Observa-se, pelos gráficos III e IV (Fig. 2) que a percentagem de dois ou mais sintomas acumulados ê visivelmente maior no grupo A do que no grupo B. O resumo da significancia estatistica da comparação, nos grupos A e B, da ocorrência dos sintomas mostrou que: DAR, C, $H, V$ e VC predominam no grupo $A$ independentemente do sexo; DS predomina no grupo A no sexo masculino; DC predomina no grupo A no sexo feminino. Para pseudoangina não foi encontrada diferença estatística. A ausência dos sintomas foi significantemente maior no grupo B, ou seja, a nån ocorrência dos sintomag referidos é maior nas crianças sem cefaléia. 


\begin{tabular}{|c|c|c|c|c|c|c|c|c|}
\hline \multirow[t]{3}{*}{ Sintoma } & \multicolumn{8}{|c|}{ Comparaçăo } \\
\hline & A & B & \multicolumn{2}{|r|}{$\sigma^{\circ}$} & \multicolumn{2}{|r|}{ \& } & \multicolumn{2}{|r|}{89} \\
\hline & $8 \times 8$ & $8 \times 9$ & A & $\times B$ & A & $\times B$ & A & $\times B$ \\
\hline DS & $\left(0^{\prime \prime} \approx 8\right)$ & $(0<9)^{\star}$ & (A & $>B)^{\star}$ & (A & $\approx 8)$ & (A & $\Leftrightarrow B$ \\
\hline DAR & $(d \approx 8)$ & $(0 \approx 9)$ & (A & $>\mathrm{B})^{\star}$ & (A & $>8)^{\star}$ & (A & $(>B)^{*}$ \\
\hline C & $(0 \approx 8)$ & $(0 \approx 9)$ & (A & $>B)^{*}$ & (A & $>B)^{\star}$ & (A & $(>B)^{\star}$ \\
\hline$H$ & $(0 \approx 9)$ & $(0 \approx 9)$ & (A & $>8)^{\star}$ & ( $A$ & $>\mathrm{B})^{\star}$ & (A & $(>\mathrm{B})^{\star}$ \\
\hline V & $(0 \approx 8)$ & $(\delta \approx 9)$ & (A & $>B)^{\star}$ & (A & $>B)^{\star}$ & (A & $(>\mathrm{B})^{\star}$ \\
\hline$D C$ & $(0 \approx 9)$ & $(0 \approx 9)$ & (A & $\approx B)$ & (A & $>\mathrm{B})^{\star}$ & (A & $(>B)^{*}$ \\
\hline VC & $(\sigma \approx q)$ & $\left(\sigma^{*} \approx 9\right)$ & (A & $>B)^{\star}$ & (A & $>B)^{\star}$ & (A & $(>B)^{*}$ \\
\hline$P$ & $(0>8)^{\star}$ & $(0 \approx 8)$ & (A & $\approx B\rangle$ & (A & $\approx B\rangle$ & (A & $\approx B\rangle$ \\
\hline
\end{tabular}

Ausência a

$\left(0^{*} \approx 9\right) \quad\left(0^{*}>9\right)^{\star} \quad(A<B)^{\star} \quad(A<B)^{\star} \quad(A<B)^{\star}$

Legenda

$$
\begin{array}{rlrl}
\delta=\text { masculino } & >\text { maior } \\
\&=\text { feminino } & < & =\text { menor } \\
\delta \&=\delta+\& & \star & =\text { significante } \\
\approx=\text { não significante } & A & =\text { grupo com enxaqueca } \\
& B & =\text { grupo sem cefaleia }
\end{array}
$$

Tabela 5 - Resumo da significancia estatistica para os dados especificos dos sintomas e ausencia dos sintomas.

\section{COMENTARIOS}

Critérios para o diagnóstico de enxaqueca em crianças permitem que se obtenham casuisticas mais uniformes. O critério de Prensky e Sommer 32 leva em conta o maior número de dados e porisso foi o escolhido neste estudo.

A importância de se estudar o antecedente familiar de enxaqueca e os sintomas que analisamos reside, no fato de que a sua presença pode contribuir para aumentar as possibilidades diagnósticas da enxaqueca na criança. $O$ caráter hereditário é importante e na literatura é referido antecedente familiar positivo em 50 a $90 \%$ dos pacientes com enxaqueca. A análise estatística de nossa casuística mostrou que o antecedente familiar, tanto de cefaléia em geral como de enxaqueca, é maior nas crianças com enxaqueca e faixa etária de 7 a 15 

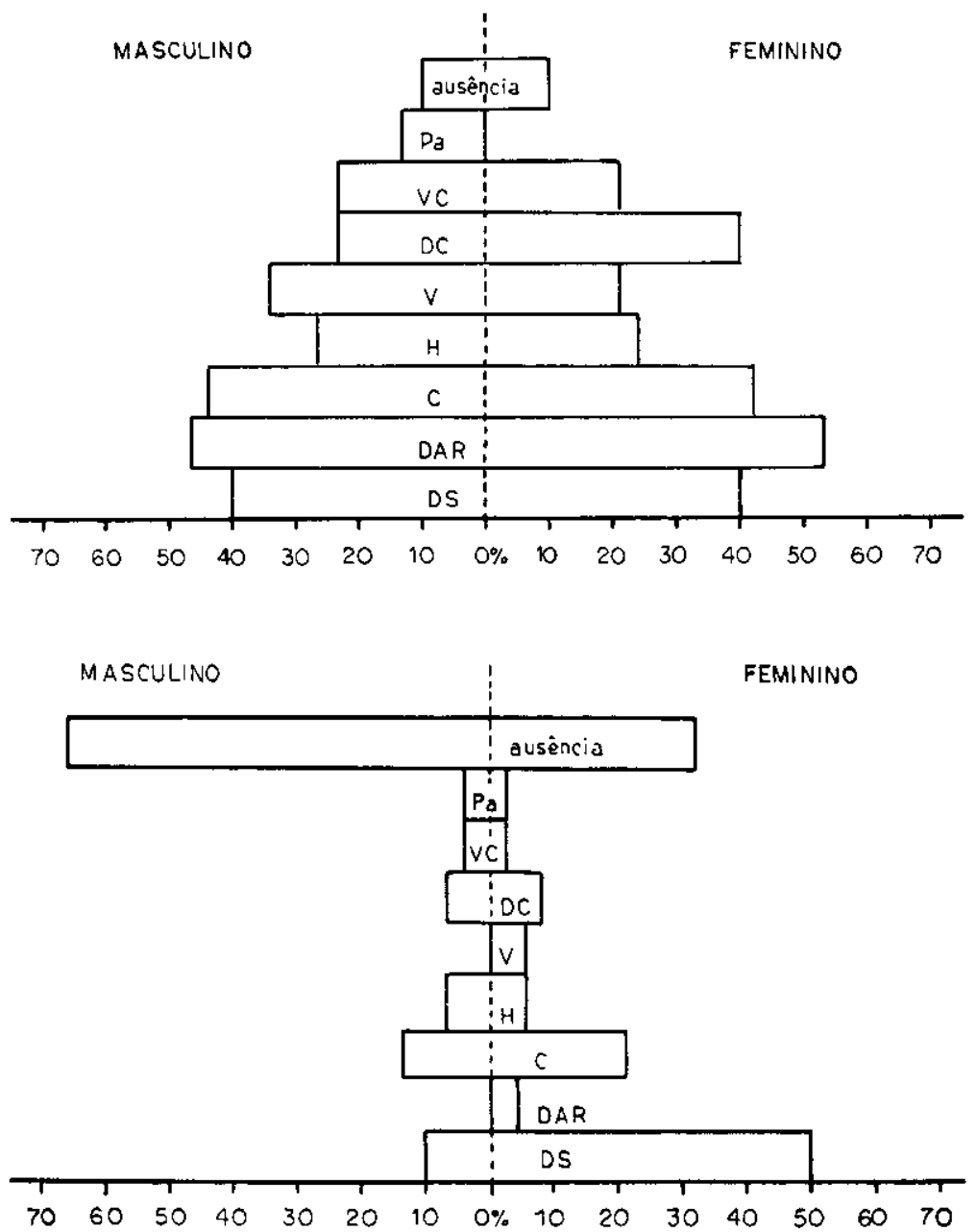

Fig. 1 - Gráficos $I$ e $I I$. Percentagem de ocorrencia tos sintomas $D S, D A R, C, H, V$, DC, VC $\mathrm{e} P$, concomitantes ou não, nos 30 casos do sexo masiculino e nos 38 do sexo feminino. Gráfico I (no alto), casos do grupo $A$, com enxaqueca. Grafico II (em baixo), casos do grupo $B$, sem cefaleia.

anos do que naquelas sem cefaléia e mesma faixa etária. A incidência de antecedente familiar de enxaqueca nos pacientes do grupo A foi semelhante à encontrada na literatura $(63,23 \%)$.

DS, DAR, C, H, V, DC, VC e P estão freqüentemente associados ao quadro da enxaqueca segundo diversos autores. Esses sintomas podem ser referidos pelos pais ou podem ser observados pelo médico, trazendo objetividade à anamnese. $O$ estudo desses sintomas tem sido feito de forma pouco regular e por critérios diversos, determinando resultados dificilmente comparáveis. Outras vezes os sintomas referidos são estudados isoladamente. Os autores estudam grupos de crianças com diagnóstico de cefaléia em geral ou especificamente com enxaqueca. A faixa etária e o grupo controle não são estabelecidos de forma uniforme. Del Bene 9 mostrou que DS, DAR, V, DC e VC predominavam no grupo de crianças 

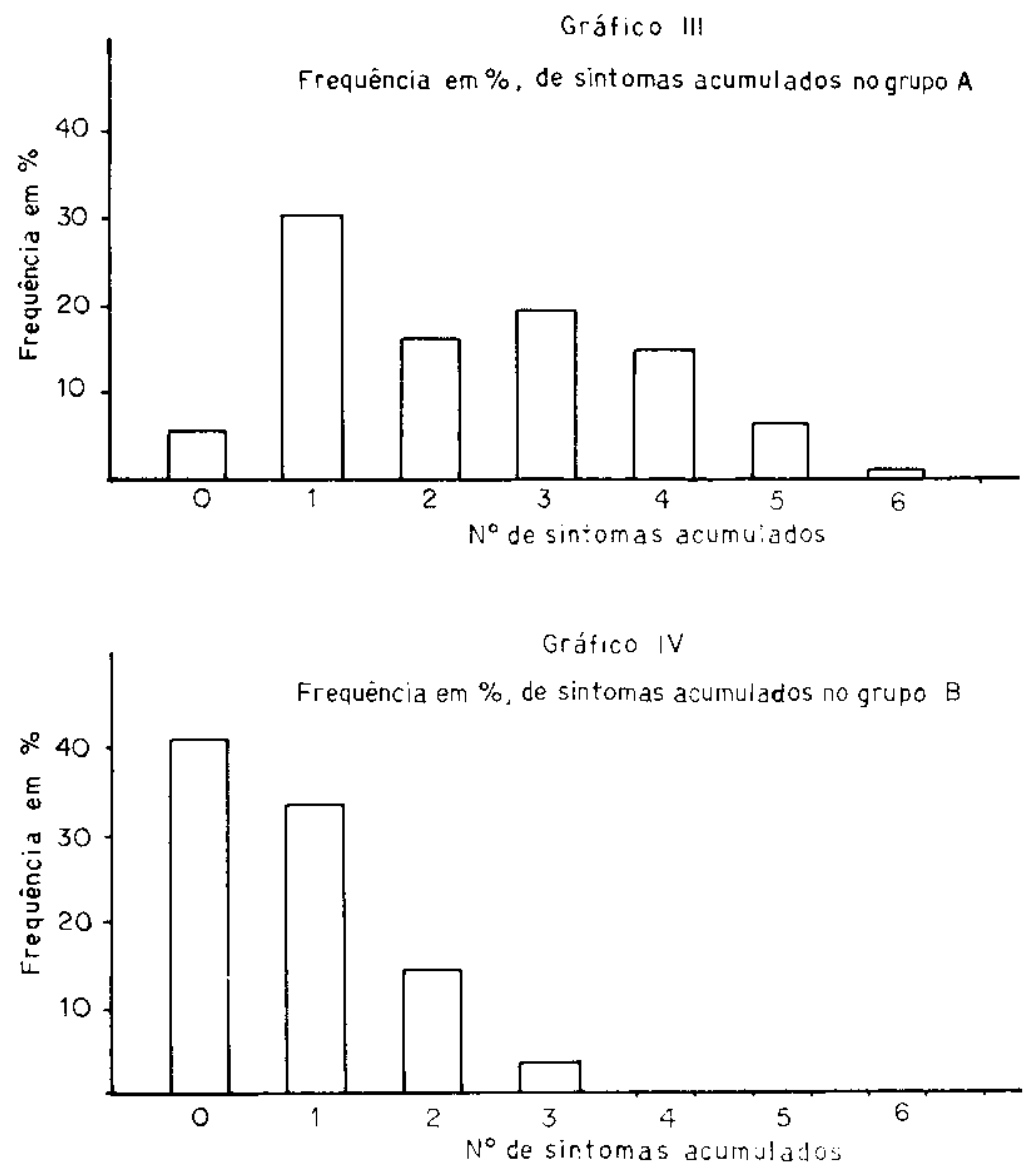

Fig. 2-Gráficos $I I I$ e IV. Freqüência em percentagem dos sintomas acumulados. Grafico $I I I$, casos do grupo $A$, com ensaqueca. Gráfico $I V$, aasos do grupo $B$, sem cefaléia.

com cefaléia em relação aos controles. H não mostrou diferença estatística. Esse autor estudou crianças com idade média de 11 anos e considerou a cefaléia e não especificamente a enxaqueca. Além disso, o grupo controle foi de crianças com e sem antecedente familiar de cefaléia. Apesar disso, o autor considerou esses sintomas associados ao antecedente familiar de cefaléia como fatores de risco de cefaléia em criança. Outros autores se referem aos sintomas enumerados como parfe do quadro clínico da enxaqueca, sem maiores contribuiçōes para o diagnóstico.

$\mathrm{Na}$ sequiência de nossos estudos vamos procurar responder a outras perguntas, tais como se os sintomas estudados incidem mais em crianças com enxaqueca que naquelas com outras patologias, como por exemplo epilepsia ou distúrbios de aprendizagem. Nesta linha de pesquisa vamos tentar estabelecer a real importância deste grupo de sintomas de modo a poder confirmá-los como fatores de risco da enxaqueca.

Em conclusão, neste trabalho mostra-se que o antecedente familiar de enxaqueca e ou de cefaléia em geral e dor abdominal recorrente, cinetose, vertigens, hiperatividade, vômitos cíclicos, em ambos os sexos, distúrbio de sono 
no sexo masculino, dores do crescimento no sexo feminino, incidem mais nas crianças com enxaqueca na faixa etária de 7 a 15 anos, em comparação com crianças sem cefaléia e pareadas por idade e sexo. Pseudoangina não mostrou diferença significante.

RESUMO

A comparação de dois grupos de crianças (grupo A, com enxaqueca e grupo $\mathrm{B}$, controle, sem cefaléia) com idade de 7 a 15 anos mostrou quanto à ocorrência de distúrbio de sono, dor abdominal recorrente, cinetose, hiperatividade, vertigens, dores do crescimento, vômitos cíclicos, pseudoangina e antecedente familiar de cefaléia em geral e/ou enxaqueca que: predomina no grupo A, dor abdominal recorrente, cinetose, vertigens, hiperatividade, vômitos cíclicos, em ambos os sexos; distúrbio de sono no sexo masculino e dores do crescimento, no feminino; antecedente familiar de cefaléia em geral e/ou enxaqueca. Pseudoangina não mostrou diferença significante. Discute-se a possibilidade de serem considerados esses fatores como fatores de risco para o diagnóstico de enxaqueca.

\section{SUMMARY}

\section{Migraine risk factors in school children.}

The occurence of sleep troubles, recurrent abdominal pain, motion sickness, hyperactivity, dizziness, limb pain, cyclic vomiting, pseudoangine and the headache or migraine family history have been studied in 68 children migraine sufferers and compared to 68 non-headache sufferers whose ages range from 7 to 15 . Data have revelead a significant predominance of those symptoms and family histories in migraine sufferers except pseudoangine which has had no significance, sleep troubles significant only in males and limb pains in females. The possibility of considering those factores as migraine risk factors is discussed.

\section{REFERENCIAS}

1. BARABAS, G.; MATHEWS, W.S. \& FERRARI, M. - Childhood migraine and motion sickness. Pediatrics $72: 188,1983$.

2. BAROLIN, G.S. - Headache in children. Adv. Neurol. 33:183, 1982.

3. BERGAMINI, G.; GUIDETTI, G. \& DALLARI, S. - La vertigine nella sindrome ricorrente infantile: considerazioni e ipotesi eziopatogenetiche a proposito de 14 casi. Minerva pediat, $34: 577,1982$.

4. BILLE, B. - Migraine in school children. Acta paediat. (Uppsala) 51:1, 1962.

5. BLITZTEN, N.L. \& BRAMS, W.A. $\longrightarrow$ Migraine with abdominal equivalent, J. amer. med. Assoc. $86: 675,1926$.

6. BROWN, J.K. - Migraine and migraine equivalent in children. Develop. Med. Child Neurol, 19:683, 1977.

7. CHAO, D.; SEXTON, J.A. \& DAVIS, S.D. - Convulsive equivalent syndrome of childhood. J. Pediat. 64:499, 1964.

8. DALSGAARD-NIELSEN, T. Migraine and heredity. Acta. neurol. scand. 41: 287, 1965.

9. DEL BENE, E. - Multiple aspects of headache risk in children. Adv. Neurol. $33: 187,1982$.

10. EVIATAR, L. \& EVIATAR, A. - Vertigo in childhood. Clin. Pediat. 13:940, 1974.

11. F'ENICHEL, G.M. - Migraine as a cause of benign paroxismal vertigo of childhood. J. Pediat. $71: 114,1967$.

12. GENTILLI, P.; MARTINI, A.; COZZI, F. \& LAZZARI, R. - I dolori abdominali ricorrenti nel bambino. Minerva pediat. $35: 45,1983$.

13. GREEN, M. - Dingnosis and treatment: psycogenic, recurrent, abdominal pain. Pediatrics 40:84, 1967. 
14. GUIDETTI, G.; BERGAMINI, G. \& DALlaRI, S. - Vertigine e cefalea nell' infanzia: considerazione a proposito di tre casi. Minerva pediat. 33:587, 1981.

15. HUGHES, M.C. \& ZIMIN, R. - Children with psychogenic abdominal pains and their families: manegement during hospitalization. Clin. Pediat. 17:569, 1978.

16. JAY, G.W. - Epilepsy, migraine, and EEG abnormalities in children: a review and hypothesis. Headache $22: 110,1982$.

17. KOEHLER, B. - Benign paroxismal vertigo of childhood, a migraine equivalent. Eur. J. Pediat. 134:149, 1980.

18. KURITZKY, A.; ZIEGLER, D.K. \& HASSANEIN, R. - Vertigo, motion sickness and migraine. Headache 21:227, 1981.

19. LANZI, G.; BALONTTIN, U.; FAZZI, E. \& ROSANO, F.B. - The periodic syndrome in pediatric migraine sufferers. Cephalalgia (Suppl. 1):91, 1983.

20. LIEBMAN, W.M. - Recurrent abdominal pain in children: a retrospective survey of 119 patients. Clin. Pediat. 17:149, 1978.

21. LIVINSTON, S. - Abdominal pain as a manifestation of epilepsy. J. Pediat. $38: 687,1951$.

22. LUNDBERG, P.O. - Abdominal pain, diagnosis and therapy. Headache 15:122, 1974 .

23. MILLIC'HAP, J.G. - Cyclic vomiting as a form of epilepsy in children. Pediatrics $15: 705,1955$.

24. MIRA, E. ; PIACENTINO, G.; LANZI, G.; BALOTTIN, U. \& FAZZI, F. - Benign paroxismal vertigo in childhood: a migraine equivalent. ORL J. Otorhinolaryng. relat. Spec, 46:97, 1984 .

25. MiTCHELL, W.G.; GREENWOOD, R.S. \& MESSENHEIMER, J.A. -- Abdominal epilepsy: cyclic vomiting as the major symptom of simple partial seizures. Arch. Neurol. 40:251, 1983.

26. MOORE, M.T. - Abdominal epilepsy versus abdominal migraine. Ann. int. Med. $33: 122,1950$.

27. MORETTI, G.; MANZONI, G.C.: CAFFARRA, P. \& PARMA, M. -- Benign recurrent vertigo and its connection with migraine. Headache 20:344, 1980.

28. MULDER, D.W. - Visceral epilepsy. Arch. int. Med. 93:481, 1954.

29. NAEYE, R.L. - Cyclic fever, abdominal pain and grand mal seizure: case report. Ann, int. Med, 48:859, 1958.

30. OSTER, J. - Recurrent abdominal pain, headache and limb pains in children and adolescents. Pediatrics 50:429, 1972.

31. PRENSKY, A.L. - Migraine and migranous variants in pediatric patients. Pediat. Chin. N. Amer. 23:461, 1976.

32. PRENSKY, A.L. \& SOMMER. D. - Diagnosis and treatment of migraine in children. Neurology 29:506, 1979.

33. SIEGEL, S. - Estadistica No Paramétrica Aplicada a las Ciencias de la Conduta. Trillas, México, 1975 , p. 346 .

34. SLATER, R. - Benign recurrent vertigo. J. Neurol, Neurosurg. Psychiat. 42:363, 1979 .

35. VERCELLETTO, P.; CLER, J.M. \& FRIOL, M. - Vertiges et migraine basilaire - place, nosologique des vertiges benins de l'enfant. Rev. Oto-Neuro-Ophtal. $51: 231,1979$.

36. WATERS, W.E. - Migraine and symptomas in childhood: bilious attacks: travel sickness and eczema. Headache 12:55, 1972.

37. WATSON, P. \& STEELE, J.C. - Paroxismal dysequilibrium in the migraine syndrome of childhood. Arch. Otolaryng. 99:177, 1974.

Run dos Otonis 880, apt. 82 - 04025 - São Paulo, SP - Brasil. 